\title{
Giant Shapiro Steps in a Superconducting Network of Nanoscale $\mathrm{Nb}$ Islands
}

\author{
Martijn Lankhorst ${ }^{1} \cdot$ Nicola Poccia $^{1,2}$
}

Received: 9 October 2015 / Accepted: 19 November 2015 / Published online: 18 January 2016

(C) The Author(s) 2016. This article is published with open access at Springerlink.com

\begin{abstract}
Recently, a dynamic vortex Mott transition has been observed in an array of superconducting nanodots. Here, we report the effect of the interaction of microwave radiation on this system and we show the occurrence of giant Shapiro steps.
\end{abstract}

Keywords Vortex lattices - Superconducting proximity networks $\cdot$ Far from equilibrium phenomena $\cdot$ Microwave radiation $\cdot$ Dynamic vortex Mott transitions

\section{Introduction}

It is known that regular square arrays of BCS-type superconductors in the shape of crosses [1,2], dots [3], or anti-dots $[4,5]$ on top of both metallic and insulating substrates have been investigated on varied length scales. In particular, the zero-temperature phase can be tuned to a conductor [3] and an insulator [5] by making the distance between grains larger than the coherence length. The increased level of control over the spatial complexity and disorder in a regular square array of nanostructured superconductors has made

Nicola Poccia

nicola.poccia@utwente.nl

nicola.poccia@nano.cnr.it

1 MESA+ Institute for Nanotechnology, University of Twente, P.O. Box 217, 7500AE Enschede, Netherlands

2 NEST Istituto Nanoscienze-CNR \& Scuola Normale Superiore, Pisa, Italy possible the observation of a vortex Mott insulator-to-metal transition which occurs far from equilibrium [6]. In this paper, we measure the influence of an external RF source. We observe the well-known Shapiro steps in a sample where also the dynamic vortex Mott transition has been observed.

\section{Superconductivity and Microwave Radiation}

Superconductors interact with radiation in numerous ways. Radiation can break up Cooper pairs if the photon energy exceeds the superconducting gap, causing the resistance to increase. Furthermore, in cuprate thin films, an argon laser can decrease the resistance of the film, which persists for a long time after the laser is turned off (called persistent photoconductivity). This effect is possibly caused by the ordering of mobile charge carriers or defects [7]. In particular, when a Josephson junction is irradiated with a radio wave source, steps are created in the voltage-current characteristics. These steps are called Shapiro steps and are a direct consequence of the induced AC current in the junction. The average DC voltage as a function of current shows horizontal plateaus at integer multiples of the voltage $V_{\mathrm{SS}}=\hbar \omega /(2 e)$, called Shapiro steps [8]. When a large number of identical junctions is connected in series, they will all resonate at the same current resulting in a voltage plateau at exactly $N^{*} V_{\mathrm{SS}}$. These steps are called giant Shapiro steps [1,9] and can be used as voltage standards [10]. Moreover, they have been observed in cuprates [11] and disordered arrays [12]. Conversely, when a DC current larger than $I_{\mathrm{c}}$ is passed through a Josephson junction, it will start to emit radiation of a fixed wavelength. In an array of Josephson junctions, the collective emission can be used to create a lasing mechanism [13]. The sensitivity 

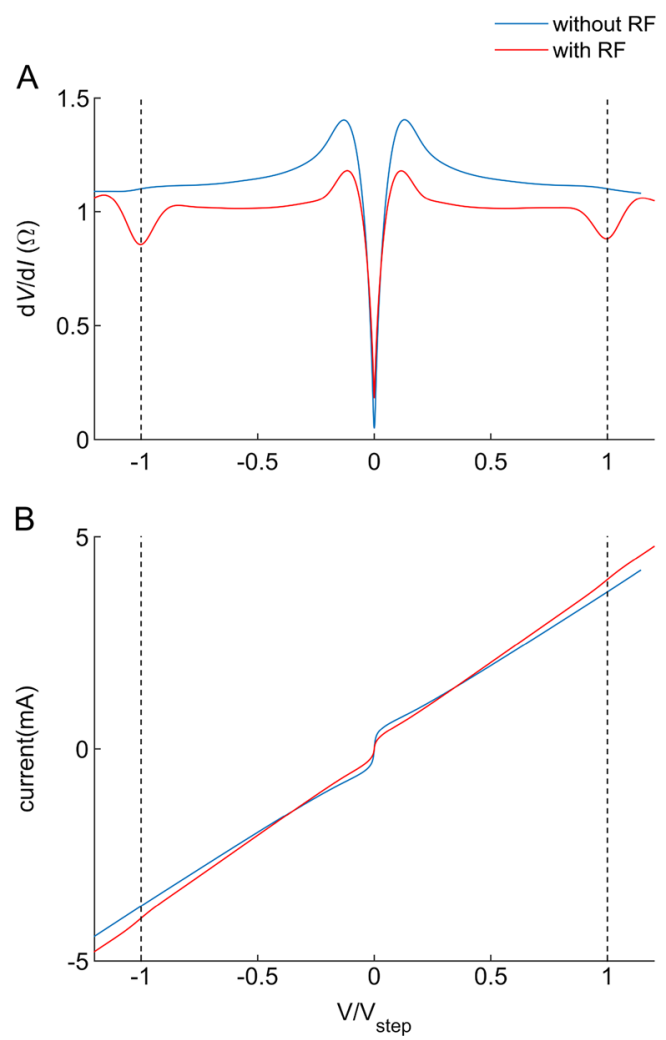

Fig. 1 IV characteristics of a square Josephson junction array of 300by-300 $\mathrm{Nb}$ dots with and without an external RF power source with a frequency of $6282 \mathrm{MHz}$. A giant Shapiro step is observed at a voltage of $3.90 \mathrm{mV}$, corresponding to the theoretical value of $N \Phi_{0} \omega /(2 \pi)$. The measurement is done at a temperature of $4.2 \mathrm{~K}$. The Shapiro step is hard to observe in the IV curve, but in the differential resistance, it can be clearly seen

to light in a broad range of frequencies has lead to numerous applications. The microwave sensitivity is applied in technologies like multiplexers, cryogenic receivers, delay lines, and antennas [14-16], while the infrared sensitivity is used in for space detectors [17]. In the search of potential applications for modern electronics, here we report an observation of giant Shapiro steps in the artificially made superconducting nanostructures with controlled complexity.

\section{Design}

Our choice to manufacture an array of proximity-coupled superconducting nanodots has been motivated by the recent interest in superconducting networks [18] and on the concept of the vortex-quantum particle mapping, which was used as a tool to explain the vortex Mott transition [6, 21].

\section{Experiment}

A square array of 300-by-300 $\mathrm{Nb}$ islands was therefore grown on a thin layer of gold on top of a $\mathrm{Si} / \mathrm{SiO}_{2}$ substrate. The substrate consists of a $6-\mu \mathrm{m}$-thick oxidized layer on p-doped Si. The gold layer was fabricated with photolithography and $\mathrm{DC}$ sputtering. The $\mathrm{Nb}$ islands were fabricated with e-beam lithography and DC sputtering. The islands have a diameter of $210 \pm 10 \mathrm{~nm}$ and the array has a lattice constant of $250 \mathrm{~nm}$. On both sides of the array, a Nb bar was deposited to ensure a uniform current through the array. A four-point probe measurement was done, but due to the equipotential nature of the $\mathrm{Nb}$ bars, this results in an effective two-point measurement between the bars. A small pickup loop was held directly above the array to apply RF radiation. A resonant frequency was found at $6282 \mathrm{MHz}$ at which all measurements are done. Furthermore, a transverse magnetic field was applied. Transport measurements were done in a liquid helium bath cryostat.
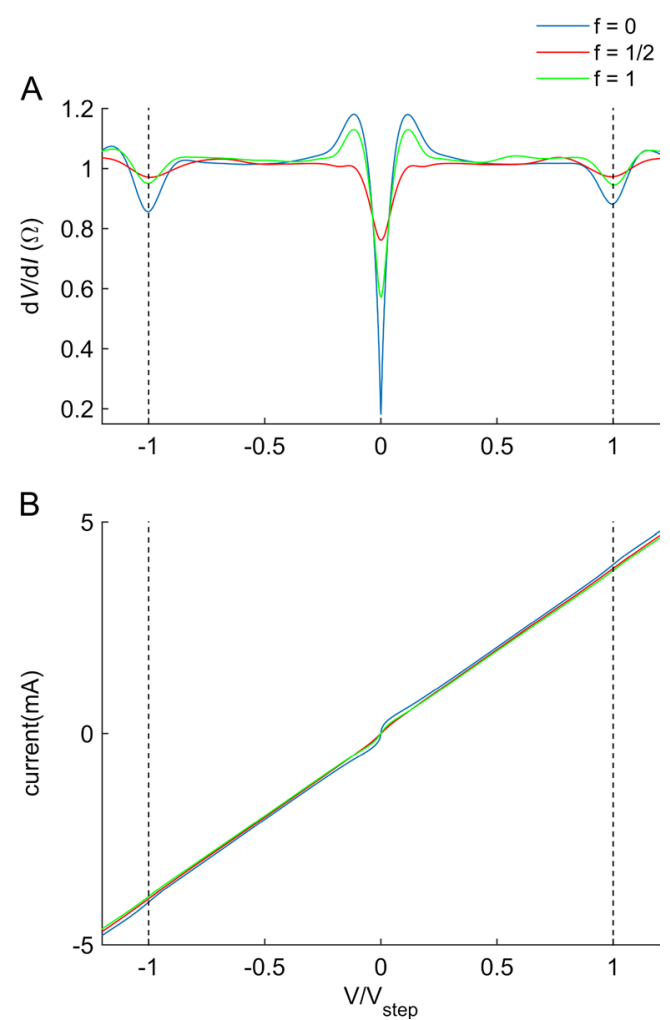

Fig. 2 IV characteristics of a square Josephson junction array of 300by-300 $\mathrm{Nb}$ islands with an external RF power source with a frequency of $6282 \mathrm{MHz}$ at various magnetic fields $f=B A / \Phi_{0}$. The shape of the Shapiro step is dependent on the magnetic field 


\section{Results and Discussion}

Figure 1 shows the voltage measurement on the array with (blue) and without (red) an external RF field. From the IV curve, it is hard to see the plateau, but by taking the derivative $\mathrm{dV} / \mathrm{dI}$, one observes a dip of about $20 \%$ precisely at the expected voltage $V_{\mathrm{SS}}=N \Phi_{0} \omega /(2 \pi)=3.90 \mathrm{mV}$. Here, $N$ is the number of junctions, $\Phi_{0}$ is the flux quantum, and $\omega$ is the angular frequency of the incoming radiation. The dip is clearly not present in the unirradiated case. The depression in $\mathrm{dV} / \mathrm{dI}$ is smaller than in similar studies of regular square arrays $[1,2,4]$, but a direct comparison is difficult because in this setup, it is unknown how much power the pickup loop actually converts into radiation. Figure 2 shows transport measurements with constant RF radiation for different magnetic fields. The magnetic field is normalized to $B_{0}=\Phi_{0} a^{2}$ where $a^{2}$ is the area of one cell of the lattice. At $B=B_{0}$, the depression of the $\mathrm{dV} / \mathrm{dI}$ at the Shapiro step is smaller than without a magnetic field, but at $f=1 / 2$, it is smaller still. Fractional Shapiro steps observed in previous studies $[1,2,4]$ are not clearly visible. The reason for this could be that the coupled power of the RF radiation is too small.

The magnetic field $B_{0}=33.1 \mathrm{mT}$ is significantly smaller than the $B_{\mathrm{c} 1}$ value of the bulk Nb $(\sim 100 \mathrm{mT}$ at $4.2 \mathrm{~K})$ [16]. It is expected that in our sample, $B_{\mathrm{c} 1}$ for both the bars and the dots will be larger than $B_{0}$, such that the sample will contain Josephson vortices, but no Abrikosov vortices. At $f=1$, all elementary squares with side $a$ are filled with a Josephson vortex. At $f=1$, the vortices exactly counter the external magnetic field, which leads to a homogeneous current through the device (no current goes through the transverse junctions). This is the same for $f=0$, and indeed the $\mathrm{dV} / \mathrm{dI}(\mathrm{I})$ curve looks the same for $f=1$ and $f=0$. For $f$ $=1 / 2$, however, the vortices counter the mean external field, but the local difference causes a ground state of circulating clockwise and counter-clockwise currents in a checkerboard configuration [17].

The depression in the differential resistance at the first Shapiro step at $f=1 / 2$ is smaller than that at $f=1$, which indicates that there is some interplay between the vortices and the RF radiation. Possibly, the vortices distort the otherwise uniform current preventing the synchronization necessary for giant Shapiro steps.

\section{Conclusions}

We have observed giant Shapiro steps in a sample consisting of a superconducting array of dots where we also observe the dynamic vortex Mott transition. With the increasing precision and control of a complex landscape for superconductivity in arrays of superconducting nanostructures, complex superconducting networks will be realized by mimicking the one observed in high-temperature superconductivity [22-34] and discussed in several recent theoretical works [35-41].

Acknowledgments We thank G. Huitenga and J. Verschuur for their support during the experiments. We thank Dick Veldhuis, Frank Roesthuis and Hans Hilgenkamp for valuable discussions. The work was supported by the Dutch FOM, the NWO foundations, by the Italian Ministry of Education and Research (MIUR) and by the Marie Curie Intra-European Fellowship.

Open Access This article is distributed under the terms of the Creative Commons Attribution 4.0 International License (http:// creativecommons.org/licenses/by/4.0/), which permits unrestricted use, distribution, and reproduction in any medium, provided you give appropriate credit to the original author(s) and the source, provide a link to the Creative Commons license, and indicate if changes were made.

\section{References}

1. Lee, H.C., Newrock, R.S., Mast, D.B., Hebboul, S.E., Garland, J.C., Lobb, C.J.: Phys. Rev. B 44, 921 (1991)

2. Benz, S.P., Rzchowski, M.S., Tinkham, M., Lobb, C.J.: Phys. Rev. Lett. 64, 693 (1990)

3. Eley, S., Gopalakrishnan, S., Goldbart, P.M., Mason, N.: Nat. Phys. 8, 59 (2011)

4. Van Look, L., Rosseel, E., Van Bael, M.J., Temst, K., Moshchalkov, V.V., Bruynseraede, Y.: Phys. Rev. B 60, R6998 (1999)

5. Baturina, T.I., Vinokur, V.M., Yu, Chtchelkatchev, N.M., Nasimov, D.A., Latyshev, A.V.: EPL. Europhys. Lett., 47002+ (2011)

6. Poccia, N., Baturina, T.I., Coneri, F., Molenaar, C.G., Wang, X.R., Bianconi, G., Brinkman, A., Hilgenkamp, H., Golubov, A.A., Vinokur, V.M.: Science 349, 1202 (2015)

7. Hoffmann, A., Hasenm, J., Ledermanm, D., Schullerm, I.K., Bruynseraede, Y., Endo, T.: J. Alloys Compd. 251, 87 (1997)

8. Shapiro, S.: Phys. Rev. Lett. 11, 80 (1963)

9. Octavio, M., Free, J.U., Benz, S.P., Newrock, R.S., Mast, D.B., Lobb, C.J.: Phys. Rev. B 44, 4601 (1991)

10. Burroughs, C.J., Bent, S.P., Harvey, T.E., Hamilton, C.A.: Applied Superconductivity. IEEE Transactions on 9, 4145 (1999)

11. Cybart, S., Chen, K., Dynes, R.C.: Applied Superconductivity. IEEE Transactions on 15, 241 (2005)

12. Ravindran, K., Gómez, L.B., Li, R.R., Herbert, S.T., Lukens, P., Jun, Y., Elhamri, S., Newrock, R.S., Mast, D.B.: Phys. Rev. B 53, 5141 (1996)

13. Barbara, P., Cawthorne, A.B., Shitov, S.V., Lobb, C.J.: Phys. Rev. Lett. 82, 1963 (1999)

14. Luine, J., Abelson, L., Brundrett, D., Burch, J., Dantsker, E., Hummer, K., Kerber, G., Wire, M., Yokoyama, K., Bowling, D., et al.: Applied Superconductivity. IEEE Transactions on 9, 4141 (1999)

15. Grimes, C.C., Richards, P.L., Shapiro, S.: J. Appl. Phys. 39, 3905 (1968) 
16. Finnemore, D.K., Stromberg, T.F., Swenson, C.A.: Phys. Rev. 149, 231-243 (1966)

17. Teitel, S., Jayaprakash, C.: Phys. Rev. Lett. 51, 1999-2002 (1983)

18. Bianconi, G.: Phys. Rev. E 85, 061113+ (2012)

19. Feynman, R.P., Leighton, R.B., Sands, M.: The New Millennium Edition: Quantum Mechanics (Volume 2) Basic Books: The Feynman Lectures on Physics, vol. III (2011)

20. Sondhi, S.L., Shahar, D.: Rev. Mod. Phys. 69, 315 (1997)

21. Nelson, D., Vinokur, V.: Phys. Rev. B 48, 13060 (1993)

22. Fratini, M., Poccia, N., Ricci, A., Campi, G., Burghammer, M., Aeppli, G., Bianconi, A.: Nature 466, 841 (2010)

23. Poccia, N., Ricci, A., Campi, G., Fratini, M., Puri, A., Di Gioacchino, D., Marcelli, A., Reynolds, M., Burghammer, M., Saini, N.L., et al.: Proc. Natl. Acad. Sci. 109, 15685 (2012)

24. Poccia, N., Ricci, A., Bianconi, A.: J. Supercond. Nov. Magn. 24, 1195 (2011)

25. Ricci, A., Poccia, N., Campi, G., Joseph, B., Arrighetti, G., Barba, L., Reynolds, M., Burghammer, M., Takeya, H., Mizuguchi, Y., et al.: Phys. Rev. B 84, 060511+ (2011)

26. Ricci, A., Poccia, N., Campi, G., Coneri, F., Caporale, A.S., Innocenti, D., Burghammer, M., Zimmermann, M., Bianconi, A.: Sci. Rep., 3 (2013)

27. Campi, G., Ricci, A., Poccia, N., Barba, L., Arrighetti, G., Burghammer, M., Caporale, A.S., Bianconi, A.: Phys. Rev. B 87, $014517+(2013)$

28. Poccia, N., Chorro, M., Ricci, A., Xu, W., Marcelli, A., Campi, G., Bianconi, A.: Appl. Phys. Lett. 104, 221903+ (2014)
29. Ricci, A., Poccia, N., Campi, G., Coneri, F., Barba, L., Arrighetti, G., Polentarutti, M., Burghammer, M., Sprung, M., Zimmermann, M.V., et al.: New J. Phys. 16, 053030+ (2014)

30. Poccia, N., Ricci, A., Campi, G., Caporale, A.S., Bianconi, A.: J. Supercond. Nov. Magn. 26, 2703 (2013)

31. Campi, G., Ricci, A., Poccia, N., Bianconi, A.: J. Supercond. Nov. Magn. 27, 987 (2014)

32. Ricci, A., Poccia, N., Joseph, B., Innocenti, D., Campi, G., Zozulya, A., Westermeier, F., Schavkan, A., Coneri, F., Bianconi, A., et al.: Phys. Rev. B, 91 (2015)

33. Poccia, N., Campi, G., Fratini, M., Ricci, A., Saini, N.L., Bianconi, A.: Phys. Rev. B 84, 100504+ (2011)

34. Campi, G., Bianconi, A., Poccia, N., Bianconi, G., Barba, L., Arrighetti, G., Innocenti, D., Karpinski, J., Zhigadlo, N.D., Kazakov, S.M., et al.: Nature 525, 359 (2015)

35. Phillabaum, B., Carlson, E.W., Dahmen, K.A.: Nat. Commun. 3, $915+(2012)$

36. Tahir-Kheli, J.: New J. Phys. 15, 073020+ (2013)

37. Saarela, M., Kusmartsev, F.V.J.: Supercond. Nov. Magn. 28, 1337-1341 (2015)

38. Kugel, K., Rakhmanov, A., Sboychakov, A., Poccia, N., Bianconi, A.: Phys. Rev. B 78, 165124+ (2008)

39. Bianconi, A., Poccia, N., Sboychakov, A.O., Rakhmanov, A.L., Kugel, K.I.: Supercond. Sci. Technol. 28, 024005+ (2015)

40. de Mello, E.V.L.: J. Supercond. Nov. Magn. 25, 1347 (2012)

41. de Mello, E.V.L., Sonier, J.E.: J. Phys. Condens. Matter 26, $492201+(2014)$ 